\title{
Comparative proteomic analysis form patients with schizophrenia and bipolar disorder
}

\author{
L.P. Smirnova ${ }^{1 *}$, E.M. Dmitrieva ${ }^{1}$, A.A. Letova ${ }^{2}$, A.A. Seregin ${ }^{1}$, G.G. Simutkin ${ }^{1}$, \\ A.V. Semke ${ }^{1}$, S.A. Ivanova ${ }^{1}$ \\ ${ }^{1}$ Mental Health Research Institute, Tomsk National Research Medical Center of the Russian Academy \\ of Sciences, Tomsk, Russia \\ ${ }^{2}$ Siberian State Medical University, Tomsk, Russia \\ *e-mail:lpsmirnova@yandex.ru
}

Key words: schizophrenia, sipolar disorder, protein markers, proteomics, blood serum

Motivation and Aim: Schizophrenia and bipolar disorder (BD) are the most important mental disorders for social life. They represent a heterogeneous group of endogeneous mental disorders with unclarified ethiology and pathophysiological mechanisms at present. Since diagnostics of mental disorders is based only on clinical symptoms, there is a necessity in development of additional methods of biochemical/paraclinical diagnostics. The search for blood based biomarkers which may be used for diagnostics and prognosis of therapy efficacy are very important presently. Purpose of study is revealing significant differences in serum proteomes in schizophrenia, BD and matched healthy controls.

Methods and Algorithms: The sample preparation included affinity removing of six major proteins, separation by $1 \mathrm{D}$ electrophoresis, in-gel tryptic hydrolysis, and LC-MS/ MS peptide analysis using LTQ Orbitrap Velos mass spectrometer.

Results: When comparing proteome profiles, different unique protein sets were revealed (absent in other groups): 22 proteins typical for schizophrenia, and 20 - for BD. Protein set in schizophrenia was mostly associated with nucleic acid and protein metbolism, immune response, cell communication, and cell growth and maintenance. Protein set in $\mathrm{BD}$ was mostly associated with cell growth and maintenance, nucleic acid metabolism regulation, immune response, protein metabolism, transport and cell communication. Concentrations of ankyrin repeat domain-containing protein 12 (ANKRD12), coagulation factor XIII, and cadherin 5 in serum samples were determined by ELISA. Significant difference between three groups was revealed in ANKRD12 concentration $(p=0.02)$, with maximum elevation of ANKRD12 concentration (mediana level) in schizophrenia followed by BD. Cadherin 5 concentration differed significantly $(p=0.035)$ between schizophrenic patients with prevailing positive symptoms and those with prevailig negative symptoms.

Conclusion: Our results are presumably useful for discovering the new pathways involved in endogeneous psychotic disorders.

Acknowledgements: Mass spectrometric analysis was carried out using the equipment of "Human Proteome" Core Facility of the Institute of Biomedical Chemistry (IBMC) Moscow which is supported by Ministry of Education and Science of the Russian Federation (unique project ID RFMEFI62117X0017). Support by Grant of RSF No. 18-15-00053. 